\title{
EFEITO DA VARIAÇÃO DA TEMPERATURA SOBRE O DESENVOLVIMENTO EMBRIONÁRIO E PÓS-EMBRIONÁRIO DE CHRYSOPERLA RAIMUNDOI FREITAS \& PENNY (NEUROPTERA: CHRYSOPIDAE)
}

\author{
L.G.A. Pessoa ${ }^{1}$, S. de Freitas ${ }^{2}$, E. de S. Loureiro ${ }^{3}$
}

${ }^{1}$ Universidade Federal de Mato Grosso do Sul, CP 112, CEP 79560-000, Chapadão do Sul, MS, Brasil. E-mail: gugamorim@yahoo.com.br

\section{RESUMO}

\begin{abstract}
Objetivou-se verificar o efeito da variação da temperatura sobre as diferentes fases de desenvolvimento de Chrysoperla raimundoi Freitas \& Penny, nas temperaturas de 19, 22, 25, 28 e $31^{\circ}$ C. Ovos com até 24 horas de idade foram individualizados em tubos de vidro e, após a eclosão, as larvas foram alimentadas com ovos de Sitotroga cerealella Oliver. Foram avaliadas a duração e viabilidade da fase de ovo, dos ínstares e dos períodos larval, de pré-pupa, pupa e do ciclo biológico, além da determinação da temperatura base $(\mathrm{Tb})$ e da constante térmica $(\mathrm{K})$. Verificou-se que a duração das diferentes fases de desenvolvimento de C. raimundoi foi inversamente proporcional ao aumento da temperatura. De maneira geral, não houve efeito sobre a viabilidade das diferentes fases. A temperatura base variou de 8,9 a $17,1^{\circ} \mathrm{C}$ e a constante térmica de 18,25 a $335,34 \mathrm{GD}$, em função da duração de cada fase de desenvolvimento do inseto.
\end{abstract}

PALAVRAS-CHAVE: Crisopídeo, controle biológico, temperatura.

\section{ABSTRACT}

EFFECT OF TEMPERATURE VARIATION ON CHRYSOPERLA RAIMUNDOI FREITAS \& PENNY (NEUROPTERA: CHRYSOPIDAE) EMBRYONIC AND POST-EMBRYONIC DEVELOPMENT. This work was carried out to evaluate the effect of different temperatures on different Chrysoperla raimundoi Freitas \& Penny development stages in the laboratory. Eggs around $24 \mathrm{~h}$ were individualized in glass tubes and kept at 19,22, 25, 28 and $31^{\circ} \mathrm{C} ; 60 \pm 10 \mathrm{RH}$ and $12 \mathrm{~h}$ of light. The larvae were fed with Sitotroga cerealella (Oliver) eggs. Evaluations were made of the viability and the developmental time of the egg, of each instar, the larval stage, the pre-pupae and pupae and biological cycle; moreover, the base temperature $(\mathrm{Tb})$ and the thermal constants $(\mathrm{K})$ of these stages was determined. It was observed that the developmental time of C. raimundoi in different development stages decreased as the temperature increased. Generally, it did not have any effect on the viability of the different stages. The base temperature varied from 8.9 to $17.1^{\circ} \mathrm{C}$ and the thermal constants from 18.25 to $335.34 \mathrm{GD}$, in function of the duration of each phase of development of the insect.

KEY WORDS: Lacewing, biological control, temperature.

\section{INTRODUÇÃO}

Os crisopídeos são importantes predadores encontrados em vários agroecossistemas, tais como algodão, citros, milho, soja, alfafa, fumo, videira, macieira, seringueira dentre outros, associados a artrópodes-praga que apresentam incidência estacional ou não e tegumento facilmente perfurável. Os trabalhos verificando o potencial desse grupo de predadores no controle de artrópodes-praga no Brasil são relativamente recentes, destacando-seestudos de sua utilização no controle de Alabama argillacea (Hübner) (Lepidoptera:Noctuidae), dos pulgões Aphis gossypii Glover, Schizaphis graminum (Rondani) e Rhodobium porosum (Sanderson) (Hemiptera: Aphididae), algumas cochonilhas dos citros, tais como Coccus sp. (Hemiptera: Coccidae), Orthezia sp. (Hemiptera:Ortheziidae), Pinnaspis sp.e Selenaspidus

${ }^{2}$ Universidade Estadual Paulista, Faculdade de Ciências Agrárias e Veterinárias, Departamento de Entomologia, Jaboticabal, SP, Brasil.

${ }^{3}$ Universidade Federal da Grande Dourados, Faculdade de Ciências Biológicas e Ambientais, Dourados, MS, Brasil. 
sp.(Hemiptera: Diaspididae), edo percevejo-de-renda da seringueira Leptopharsa heveae Drake e Poor (Hemiptera:Tingidae) (CARVALHO;SOUZA, 2000; FreITAS 2001a). A fase larval desses predadores compreende três ínstares. Após o terceiro, as larvas tecem um casuloesféricoondepassam por profundahistogênese e organogênese, formando o estágio pupal. Após alguns dias, a pupa se transforma em pupa móvel que rompe o casulo, se fixa a um substrato, onde passa pela última ecdise, surgindo o adulto(FreITAS, 2001a).

A eficiência dos inimigos naturais é afetada pelas condições ambientais, principalmente a temperatura (SAMSON; BLOOD, 1979). Esseéum dos fatores abióticos que apresenta grande influência no ciclo biológico dos insetos, tanto direta quanto indiretamente. Diretamente afeta seu desenvolvimento e seu comportamentoe, indiretamente afeta sua alimentação. Segundo CANARD; PRINCIPI (1984), a temperatura tem papel fundamental no desenvolvimento dos crisopídeos. Os efeitos desse fator sobre algumas espécies desse grupo de predadores já foram comprovados, verificando-se que a duração das diferentes fases do desenvolvimento desses predadores é inversamente proporcional ao aumento da temperatura e a velocidade diretamente proporcional a esse aumento, afetando também as viabilidades e o potencial reprodutivo (Silva, 1991; AlbuQuerque et al., 1994; Figueira et al., 2000; MAIA et al., 2000; FONSECA et al., 2001).

Dentre os vários gêneros de crisopídeos que ocorrem no Brasil, Chrysoperla Steinmann contém quatro espécies, com um grande número de atributos que as tornam predadores-chaveem muitos sistemas agrícolas (King; NordLund, 1992; Freitas, 2003). A espécie Chrysoperla raimundoi Freitas e Penny (Neuroptera: Chrysopidae) foi relatada ocorrendo em pomares de citrus e goiaba (FREITAS; PENNY, 2001), podendo estar associada a regulação das populações de pragas existentes nesses cultivos. Como sua descrição ocorreu em 2001, pouco se conhece a respeito da sua biologia. Assim, este trabalho tem o objetivo de verificar o efeito da variação da temperatura sobre o desenvolvimento embrionário e pós-embrionário dessa espécie em condições de laboratório.

\section{MATERIALEMÉTODOS}

Adultos de crisopídeos foram coletados em seringal localizado na Fazenda-Escola da Faculdade de Ciências Agrárias e Veterinárias (FCAV/UNESP), Jaboticabal, Estado de São Paulo, levados a laboratório onde, após triagem e identificação, adultos de $C$. raimundoi foram acondicionados em gaiolas decloreto de polivinila (PVC). A partir da criação-estoque, os crisopídeos foram multiplicados e criados em laboratório segundo metodologia proposta por FREITAS (2001b), sendo utilizados insetos da terceira geração para montagem dos bioensaios.

Para verificar-se o efeito da temperatura sobre o desenvolvimento deC. raimundoi, utilizaram-se ovos com até 24 horas de idade, os quais foram individualizados em tubos de vidro vedados com filme plástico. Após a eclosão, cada larva foi alimentada ad libitum com ovos de Sitotroga cerealella (Oliver) (Lepidoptera:Gelechiiidae). Os bioensaios foram conduzidos em salas climatizadas a $19 \pm 2^{\circ} \mathrm{C}, 22 \pm 2^{\circ} \mathrm{C}$, $25 \pm 2^{\circ} \mathrm{C}, 28 \pm 2^{\circ} \mathrm{Ce} 31 \pm 2^{\circ} \mathrm{C}, 60 \pm 10 \%$ de UR e fotofase de 12 horas.

Adotou-se o delineamento inteiramente casualizado, com dez repetições por tratamento, cada um constituído por três ovos oularvas deC. raimundoi, sendo avaliados a duração e viabilidade da fase de ovo, dos ínstares e dos períodos larval, de pré-pupa, pupa e do ciclo biológico. A mudança de ínstar foi constatada observando-se a presença da exúvia, a qual foi retirada com um pincel. Também foi determinado o limiar térmico inferior ( $\mathrm{Tb}$ ) ea constante térmica (K) em graus dia (GD), utilizando o método da hipérbole (HADDAD et al., 1999). Os dados referentes à viabilidade dosínstares e das fases delarva, pré-pupa e pupa foram transformados para arco-seno da (x/ $100)^{1 / 2}$. Aqueles referentes à duração dos ínstares, fase larval, fase de pré-pupa, pupa e ciclo biológico foram transformados para $(x+0,5)^{1 / 2}$. Efetuou-se a análise de variância e o teste de agrupamento de médias de Tukey a $5 \%$ de probabilidade.

\section{RESULTADOSEDISCUSSÃO}

\section{Duração das diferentes fases de desenvolvimen- to de C. raimundoi}

Houve diminuição significativa na duração de todas as fases de desenvolvimento de $C$. raimundo $i$ à medidaqueocorreuaumentonatemperatura(Tabela1). Oaumento de $3^{\circ}$ a temperatura de $19^{\circ} \mathrm{C}$ proporcionou maior redução na duração do período embrionário quando comparado às reduções verificadas nas demais temperaturas. Esses resultados demonstraram que as larvas de C. raimundoi apresentaram maior sensibilidade às temperaturas inferiores a $22^{\circ} \mathrm{C}$, evidenciando melhor adaptação dessa espécie a uma condição climática caracterizada por temperaturas médias mais elevadas, como aquelas verificadas em muitos locais da Região Neotropical, como sugerido por FigueIra et al. (2000) para a espécie C. externa (Hagen) (Neuroptera:Chrysopidae).

Comparando-secoma temperatura de $31^{\circ} \mathrm{C}$, observa-se que a de $19^{\circ} \mathrm{C}$ apresentou incremento médionos valores dos parâmetros avaliados acima dos $60 \%$ em relação às demais temperaturas testadas (Tabela 2). 
Tabela 1 - Duração em dias ( \pm EP) das fases de desenvolvimento e ciclo biológico de Chrysoperla raimundoi em cinco temperaturas. UR $=60 \pm 10 \%$, fotofase 12 horas.

\begin{tabular}{lcccccccc}
\hline Tratamento & Ovo & 1o ínstar & $2^{\circ}$ ínstar & 3o ínstar & Fase larval & Pré-pupa & Pupa & Larva - adulto \\
\hline $19^{\circ} \mathrm{C}$ & $9,0 \pm 0,08 \mathrm{~d}$ & $7,5 \pm 0,12 \mathrm{e}$ & $5,7 \pm 0,12 \mathrm{~d}$ & $6,4 \pm 0,22 \mathrm{c}$ & $19,7 \pm 0,37 \mathrm{~d}$ & $7,5 \pm 0,19 \mathrm{~d}$ & $14,7 \pm 0,28 \mathrm{e}$ & $42,6 \pm 0,82 \mathrm{e}$, \\
$22^{\circ} \mathrm{C}$ & $5,5 \pm 0,12 \mathrm{c}$ & $5,0 \pm 0,22 \mathrm{~d}$ & $3,8 \pm 0,17 \mathrm{c}$ & $3,9 \pm 0,36 \mathrm{~b}$ & $13,0 \pm 0,74 \mathrm{c}$ & $4,7 \pm 0,27 \mathrm{c}$ & $6,1 \pm 0,16 \mathrm{c}$ & $31,9 \pm 0,41 \mathrm{~d}$ \\
$25^{\circ} \mathrm{C}$ & $4,1 \pm 0,10 \mathrm{~b}$ & $3,8 \pm 0,07 \mathrm{c}$ & $3,2 \pm 0,24 \mathrm{~b}$ & $2,8 \pm 0,09 \mathrm{a}$ & $9,6 \pm 0,12 \mathrm{~b}$ & $3,7 \pm 0,20 \mathrm{~b}$ & $7,4 \pm 0,18 \mathrm{~d}$ & $25,1 \pm 0,29 \mathrm{c}$ \\
$28^{\circ} \mathrm{C}$ & $3,9 \pm 0,04 \mathrm{~b}$ & $3,3 \pm 0,10 \mathrm{~b}$ & $2,5 \pm 0,11 \mathrm{~b}$ & $3,0 \pm 0,04 \mathrm{a}$ & $8,9 \pm 0,18 \mathrm{~b}$ & $2,5 \pm 0,13 \mathrm{a}$ & $5,9 \pm 0,15 \mathrm{~b}$ & $21,1 \pm 0,20 \mathrm{~b}$ \\
$31^{\circ} \mathrm{C}$ & $2,9 \pm 0,05 \mathrm{a}$ & $2,7 \pm 0,10 \mathrm{a}$ & $1,5 \pm 0,11 \mathrm{a}$ & $2,6 \pm 0,20 \mathrm{a}$ & $6,9 \pm 0,33 \mathrm{a}$ & $2,3 \pm 0,27 \mathrm{a}$ & $5,0 \pm 0,00 \mathrm{a}$ & $16,9 \pm 0,00 \mathrm{a}$ \\
\hline $\mathrm{CV}(\%)$ & 2,40 & 4,34 & 6,74 & 7,84 & 4,97 & 7,35 & 3,08 & 1,98 \\
\hline
\end{tabular}

Médias seguidas pela mesma letra na coluna não diferem significativamente entre si pelo teste de Tukey $(\mathrm{P}<0,05)$.

Tabela 2 - Incremento médio (\%) na duração dos estádios de Chrysoperla raimundoi em função da temperatura. UR = 60 $\pm 10 \%$, fotofase 12 horas.

\begin{tabular}{lccrrrrrr}
\hline Tratamento & Ovo & 1 ínstar & 2o ínstar & 3 ínstar & Fase larval & Pré-pupa & Pupa & Larva - adulto \\
\hline $19^{\circ} \mathrm{C}$ & 67,7 & 64,0 & 73,7 & 59,4 & 65,0 & 69,3 & 66,0 & 60,3 \\
$22^{\circ} \mathrm{C}$ & 42,3 & 46,0 & 60,5 & 33,3 & 46,9 & 51,1 & 18,0 & 47,0 \\
$25^{\circ} \mathrm{C}$ & 29,3 & 28,9 & 53,1 & 7,1 & 28,1 & 37,8 & 32,4 & 32,7 \\
$28^{\circ} \mathrm{C}$ & 25,6 & 18,2 & 40,0 & 13,3 & 22,5 & 8,0 & 15,3 & 20,0 \\
$31^{\circ} \mathrm{C}$ & 0,0 & 0,0 & 0,0 & 0,0 & 0,0 & 0,0 & 0,0 & 0,0 \\
\hline
\end{tabular}

Tabela 3 - Viabilidade $( \pm \mathrm{EP})$ das fases de desenvolvimento de Chrysoperla raimundoi em cinco temperaturas. UR $=60$ $\pm 10 \%$, fotofase 12 horas.

\begin{tabular}{|c|c|c|c|c|c|c|c|}
\hline Tratamento & Ovo & $1^{\circ}$ ínstar & 2o ínstar & $3^{\text {o }}$ ínstar & Fase larval & Pré-pupa & Pupa \\
\hline $19^{\circ} \mathrm{C}$ & $92,3 \pm 7,46 \mathrm{a}$ & $98,0 \pm 5,69 a$ & $100,0 \pm 0,00 \mathrm{a}$ & $100,0 \pm 0,00 \mathrm{a}$ & $95,9 \pm 6,11 \mathrm{a}$ & $90,4 \pm 8,89 \mathrm{a}$ & $50,0 \pm 14,90 \mathrm{a}$ \\
\hline $22^{\circ} \mathrm{C}$ & $98,4 \pm 4,45 \mathrm{a}$ & $100,0 \pm 0,00 a$ & $99,6 \pm 3,34 \mathrm{a}$ & $99,3 \pm 5,00 a$ & $95,2 \pm 10,18 \mathrm{a}$ & $99,3 \pm 5,00 \mathrm{a}$ & $94,0 \pm 5,45 a$ \\
\hline $25^{\circ} \mathrm{C}$ & $99,6 \pm 3,34 \mathrm{a}$ & $100,0 \pm 0,00 \mathrm{a}$ & $100,0 \pm 0,00 \mathrm{a}$ & $96,6 \pm 5,10 a$ & $94,0 \pm 5,45 \mathrm{a}$ & $99,6 \pm 3,34 a$ & $77,0 \pm 6,93 \mathrm{a}$ \\
\hline $28^{\circ} \mathrm{C}$ & $94,0 \pm 5,45 a$ & $99,3 \pm 5,00 a$ & $99,3 \pm 5,00 a$ & $100,0 \pm 0,00 a$ & $90,4 \pm 8,89 a$ & $96,6 \pm 5,10 a$ & $82,0 \pm 7,53 \mathrm{a}$ \\
\hline $31^{\circ} \mathrm{C}$ & $96,6 \pm 5,10 \mathrm{a}$ & $98,0 \pm 5,69 a$ & $92,3 \pm 10,24 \mathrm{a}$ & $95,0 \pm 6,68 a$ & $66,0 \pm 9,22 \mathrm{a}$ & $46,7 \pm 12,80 b$ & $38,8 \pm 17,00 \mathrm{a}$ \\
\hline CV (\%) & 21,22 & 14,60 & 18,31 & 15,41 & 32,94 & 28,60 & 50,87 \\
\hline
\end{tabular}

Médias seguidas pela mesma letra na coluna não diferem significativamente entre si pelo teste de Tukey $(\mathrm{P}<0,05)$.

Albuquerque et al. (1994), Figueira et al. (2000) e FonSECA et al. (2001) verificaram que o período embrionário, a duração dos ínstares e da fase larval e das fases de pré-pupa e pupa de C. externa, mantida em condições de temperatura semelhantes às do presente estudo, também diminuíram à medida que ocorreu a elevação da temperatura. Essa mesma tendência foi observada por SILVA (1991) para as diferentes fases e estádios de Ceraeochrysa cubana (Hagen) (Neuroptera: Chrysopidae).

\section{Viabilidade das diferentes fases de desenvolvi- mento de $C$. raimundoi}

Com relação à viabilidade do período embrionário, dos ínstares, da fases de larva e de pupa, não se verificou efeito significativo das diferentes tempera- turas (Tabela 3). Apenas pré-pupas mantidas a $31^{\circ}$ $C$ apresentaram redução significativa da viabilidade em relação àquelas mantidas nas demais temperaturas. Apesar de não terem sido verificadas diferenças significativas para a redução na viabilidade da fase de pupa nos extremos de temperaturas estudadas, 19 e $31^{\circ} \mathrm{C}$, os valores obtidos para esse parâmetro (38,8 e 50,0\% respectivamente) devem ser levados em consideração uma vez que pode haver interferência no estabelecimento das gerações sucessivas em condições de campo, além da manutenção dessa espécie de crisopídeo em laboratório numa criação massiva.

Figueira et al. (2000), estudando o desenvolvimento de $C$. externa alimentada com ovos de $A$. argillacea, não verificaram diferenças entre as viabilidades para larvas mantidas em diferentes tempe- 
raturas. Trabalhando com essa mesma espécie e alimentado-a com ninfas do pulgão $S$. graminum, FONSECA et al. (2001) verificaram que os extremos de temperatura $\left(15\right.$ e $\left.30^{\circ} \mathrm{C}\right)$ promoveram as maiores reduções nas viabilidades dos ínstares e da fase larval. SILVA (1991) verificou que o período embrio-
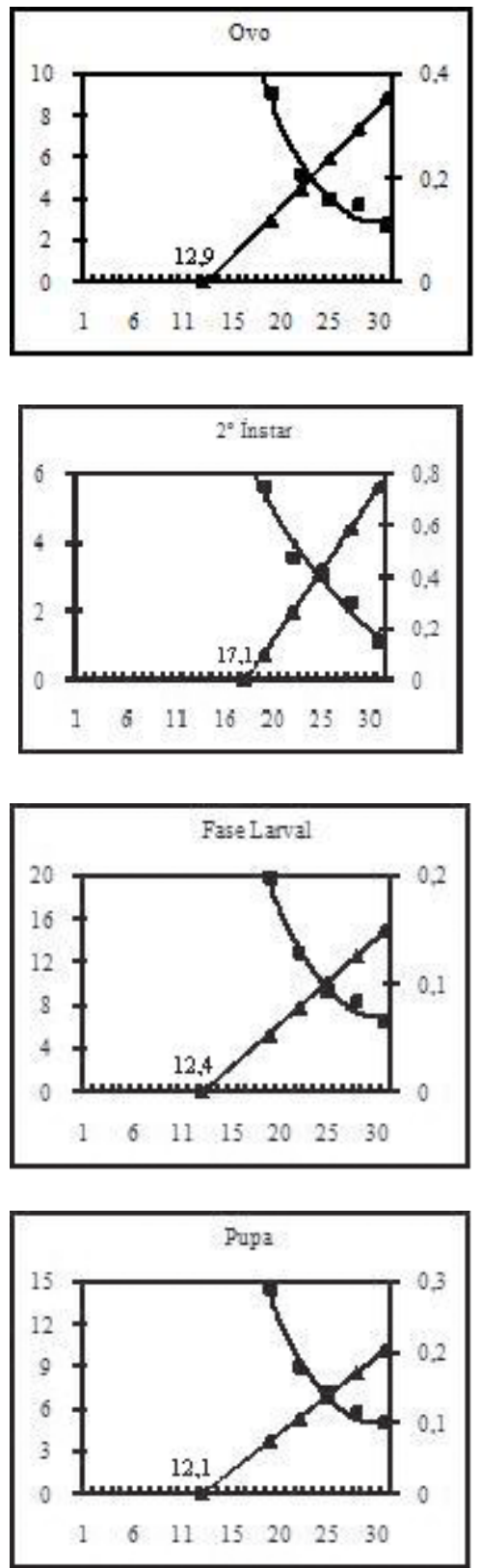

nário de C. cubana não foi afetado pelas temperaturas, porém, verificou que apenas larvas alimentadas com ovos de Anagasta kuehniella (Zeller) (Lepidoptera: Pyralidae) de primeiro e terceiro ínstares apresentaram redução na viabilidade quando mantidas na menor temperatura $\left(18^{\circ} \mathrm{C}\right)$.
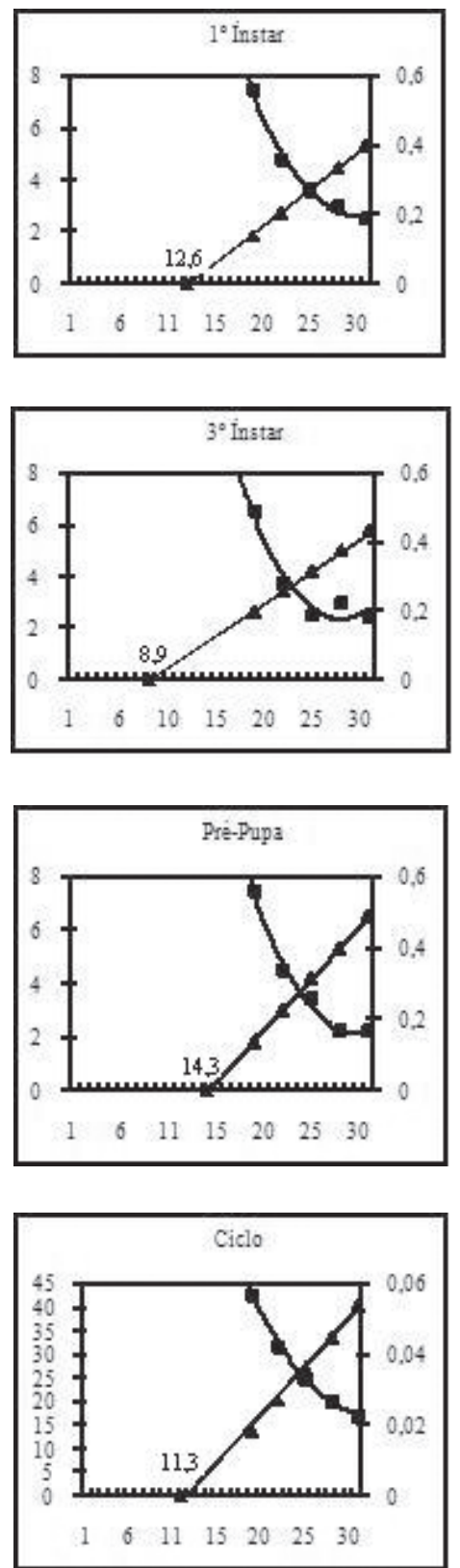

Temperatura

Veloc. de desenvolv.
Desenvolv. observado
Desenvolv. ajustado

Fig. 1 - Curvas de regressão ajustadas para a velocidade do desenvolvimento (1/D) e a duração (D) das fases imaturas de Chrysoperla raimundoi em função da temperatura. 
Tabela 4 - Temperatura-base $(\mathrm{Tb})$ e constante térmica $(\mathrm{K})$ em graus-dia (GD) para as fases do desenvolvimento de Chrysoperla raimundoi.

\begin{tabular}{lcr}
\hline Fase & $\mathrm{Tb}\left({ }^{\circ} \mathrm{C}\right)$ & $\mathrm{K}(\mathrm{GD})$ \\
\hline Ovo & 12,9 & 50,69 \\
1o ínstar $^{\circ}$ ínstar & 12,6 & 45,57 \\
3o ínstar & 17,1 & 18,25 \\
Fase larval & 8,9 & 50,96 \\
Pré-pupa & 12,4 & 124,20 \\
Pupa & 14,3 & 34,30 \\
Larva-adulto & 12,1 & 92,86 \\
\hline
\end{tabular}

Temperatura-base e constante térmica das diferentes fases de desenvolvimento de $C$. raimundo $i$

Com relação à temperatura-base $(\mathrm{Tb})$ determinada para as diferentes fases desenvolvimento dessa espécie de crisopídeo, verificou-se variação de 8,9 a 17,1 $1^{\circ} \mathrm{C}$ (Tabela 4) e (Fig. 1). Essa variação é pouco superior à encontrada por FIGUEIRA et al. (2000) e MAIA et al. (2000) para C.externa.Osmenores valores da constantetérmica (K) foram obtidosduranteo 20ínstarea fasedepré-pupa, provavelmenteem funçãoda menor duraçãoverificada para esses estádios e a menor necessidade de acúmulo deenergia. A variaçãodessa constante verificada (18,25 a 335 GD) está relacionada estritamente a duração de cadauma das fases dedesenvolvimentodeC.raimundoi.

Verifica-se que a duração das diferentes fases do desenvolvimento de C. raimundoi foi inversamente proporcional à variação da temperatura, constatando-se o papel fundamental desse fator sobre o desenvolvimentodos crisopídeos como referidopor CANARD; PRINCIPI(1984).Dessa forma, comosugeridoporSAMSON; BLOOD (1979), a eficiência dessa espécie de crisopídeo pode ser afetada pela temperatura, podendo até inviabilizar um programa de controle biológico, já que cada espécie pode apresentar respostas diferenciadas em relação à variação desse fator. Vale salientar que o conhecimento do comportamento dos inimigos naturais sob diferentes condições de temperatura podeindicar seu potencial de adaptação a uma determinada região (CIVIDANES, 2001), sendo de grande importância estudos visando a avaliação dos parâmetros biológicos desses organismosobjetivando possíveis programas de controle biológico.

\section{CONCLUSÕES}

1) A temperatura influenciou a velocidade de desenvolvimento de C. raimundoi;

2) apenas pré-pupas mantidas a $31^{\circ} \mathrm{C}$ tiveram sua viabilidade afetada;
3) as temperaturas de 25 e $28^{\circ} \mathrm{C}$ foram as mais adequadas para criação dessa espécie de crisopídeo por manter alta viabilidade e permitir o completo desenvolvimento num temposatisfatório;

4) as temperaturas-base e as constantes térmicas de C. raimundoi variaram em função do desenvolvimento do inseto.

\section{REFERÊNCIAS}

ALBUQUERQUE, G.S.; TAUBER, C.A.; TAUBER, M.J. Chrysoperla externa (Neuroptera: Chrysopidae): life history and potencial for biological control in Central and South America. Biological Control, v.4, p. 8-13, 1994.

CANARD, M.; PRINCIPI, M.M. Life histories and behavior. In: CANARD, M.; SÉMÉRIA, Y.; NEW, T.R. (Ed.). Biology of Chrysopidae. Boston: W. Junk Publishes, 1984. cap. 4, p. 57-149.

CARVALHO, C.F.; SOUZA, B. Métodos de criação e produção de crisopídeos. In: BUENO, V.H.P. (Ed.). Controle biológico de pragas: produção massal e controle de qualidade. Lavras: UFLA, 2000. cap. 6, p. 91-109.

CIVIDANES, F.J. Uso de graus-dia em entomologia, com particular referência ao controle de percevejos pragas da soja. Jaboticabal: Funep, 2001. 31p.

FIGUEIRA, L.; CARVALHO, C.F.; SOUZA. B. Biologia e exigências térmicas de Chrysoperla externa (Hagen, 1861) (Neuroptera: Chrysopidae) alimentada com ovos de Alabama argillacea (Hübner, 1818) (Lepidoptera: Noctuidae). Ciência e Agrotecnologia, v.24, n.2, p.319-326, 2000.

FONSECA, A.; CARVALHO, C.F.; SOUZA, B. Capacidade predatória e aspectos biológicos das fases imaturas de Chrysoperla externa (Hagen, 1861) (Neuroptera: Chrysopidae) alimentada com Schizaphis graminum (Rondani, 1852) (Hemiptera: Aphididae) em diferentes temperaturas. Ciência e Agrotecnologia, v.25, n.2, p.251$263,2001$.

FREITAS, S. de. O uso de crisopídeos no controle biológico de pragas. Jaboticabal: Funep, 2001a. 66p.

FREITAS, S. de. Criação de crisopídeos (bicho lixeiro) em laboratório. Jaboticabal: Funep, 2001b. 20p.

FREITAS, S. de. Chrysoperla Steinmann, 1964 (Neuroptera, Chrysopidae): descrição de uma nova espécie do Brasil. Revista Brasileira de Entomologia, v.47, n.3, p. 385-387, 2003.

FREITAS, S.; PENNY, N.D. The green lacewings (Neuroptera: Chrysopidae) of Brazilian agroecosystems. Proceedings of the California Academy of Sciences, v.52, n.19, p. 245-395, 2001. 
HADDAD, M.L.; PARRA, J.R.P.; MORAES, R.C. Métodos para estimar os limites térmicos inferior e superior de desenvolvimento dos insetos. Piracicaba: FEALQ, 1999. 29p.

KING, E.G.; NORDLUND, D.A. Propagation and augmentative releases of predators and parasitoids for control of arthropod pests. Pesquisa Agropecuária Brasileira, v.27, p.239-254, 1992.

MAIA, W.J.S.S.; CARVALHO, C.F.; SOUZA, B. Exigências térmicas de Chrysoperla externa (Hagen, 1861) (Neuroptera: Chrysopidae) alimentada com Schizaphis graminum (Rondani, 1852) (Hemiptera: Aphididae) em condições de laboratório. Ciência e Agrotecnologia, v.24, n.1, p.81-86, 2000
SAMSON, P.R.; BLOOD, P.R.B. Biology and temperature relation ships of Chrysopa sp., Micromus tasmaniae and Nabis capsiformes. Entomologia Experimentalis et Aplicatta, v.25, n.3, p. 253-259, 1979.

SILVA, R.L.X. Aspectos biológicos e determinação das exigências térmicas de Ceraeochrysa cubana (Hagen, 1861) (Neuroptera: Chrysopidae) em laboratório. 1991. 160f. Dissertação (Mestrado em Entomologia Agrícola) Universidade Federal de Lavras, Lavras, 1991.

Recebido em 17/8/07

Aceito em 16/5/09 